\title{
Human factors evaluation in VR-based shunting training
}

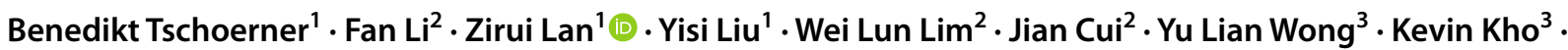 \\ Vincent Lee $^{3} \cdot$ Olga Sourina $^{1,2} \cdot$ Wolfgang Mueller-Wittig ${ }^{1,2}$
}

Accepted: 3 July 2021 / Published online: 10 August 2021

(c) The Author(s) 2021

\begin{abstract}
Shunting of trains is a task that requires meticulous adherence to all steps to guarantee safety for everyone involved during and after the procedure. These steps are currently taught using classical teaching materials, such as printouts, videos and training by experienced supervisors. However, due to limited availability of locomotives, hours for training and manpower, training of shunting operation becomes challenging in real life. In this paper, we implemented a lifelike, collaborative virtual environment for shunting training including a novel human factors evaluation system for fatigue and stress monitoring. An experiment with 12 subjects and 3 trainers has been designed and carried out to validate the usage of VR-based shunting training. Positive feedback toward the VR-based training was obtained from the subjects and trainers.
\end{abstract}

Keywords Virtual Reality $\cdot$ Human factors $\cdot$ Non-technical skills $\cdot$ Shunting $\cdot$ Training

\section{Introduction}

Shunting of engineering trains is a safety-critical activity as it involves collaborative maneuver of large trains in confined spaces, and the speed of trains has to be well controlled [1]. Various skills such as communication, decision-making, collaboration, coping with fatigue and technical knowledge are required in the shunting of engineering trains, without which, the possibility of human errors increases and is a major cause of 'crush' fatalities occurring each year in the transport industry [2]. Hence, it is essential to ensure that the operator has mastered these skills and knowledge, and that he/she became competent to conduct the shunting of engineering trains. Generally, effective training contributes significantly to the operators' competency and safety in rail industries [3].

There are numerous ways to provide training with both theory and practical elements to improve the competency of

Zirui Lan

lan.zirui@fraunhofer.sg

1 Fraunhofer Singapore, 50 Nanyang Avenue, Block NS 1, Level 5, Singapore 639798, Singapore

2 Nanyang Technological University, 50 Nanyang Avenue, Singapore 639798, Singapore

3 SMRT Train Ltd, 300 Bishan Road, Singapore 579828, Singapore both novice and experienced operators. They can be broadly divided into two types, namely on-the-job and off-the-job training [4]. On-the-job training refers to the formal training where the operators acquire specific skills during working. Off-the-job training is normally conducted in specific environments using the same or similar tools to simulate how the regular job is done. The off-the-job training can be conducted via conferences, lectures, workshop, seminars, etc. [5].

However, due to the nature of railway, training of shunting operation via either on-the-job or off-the-job way is challenging in real life. On one hand, the safety-critical concern of shunting the engineering train requires the operators to be well trained before working on-the-job. On the other hand, limited availability of locomotives, training hours and manpower decreases the efficiency of off-the-job training in real life. To address these problems and provide an alternative way for shunting training, we implemented a lifelike, collaborative virtual environment for shunting training including a novel human factors evaluation system. Virtual Reality (VR) technology has been infused into education/ training recently, as it provides a safe yet realistic environment which allows operators to undergo the training in an immersive manner. Nevertheless, VR technology has several restrictions in providing feedback to user actions, leading to impaired user experience. To provide feedback to user actions, a method was adopted to visualize user actions via 
analyzing functionality and behavior of interactive parts. Together with the VR training, the proposed human factors evaluation system can monitor the fatigue and stress level in real-time and provide an overall monitoring report about the fatigue/stress level experienced by the user, which could be used to correlate with the performance of the user during the shunting training. An experiment with 12 subjects was designed and carried out. A set of questionnaires was distributed to the subjects to get feedback about the VR-based training, and the corresponding results have been analyzed, which showed positive support for the use of VR in training shunting operations.

The rest of the paper is organized as follows: Sect. 2 presents literature review on VR-based railway operation training and human factors study in railway. In Sect. 3, the overall system design and implementation are introduced. An experiment to validate the training system and the collected user feedback are presented in Sect. 4 and Sect. 5, respectively. Section 6 concludes the study with discussions about the limitations and future works.

\section{Related works}

\subsection{VR-based railway operation training}

VR is a useful tool that enables railway operators to receive training in a safe yet realistic environment, learning the skills necessary to conduct operations in the real world. Much effort goes into creating credible mechanics that simulate the real world closely. Guan et al. [6] described how traction and braking operations can be mathematically simulated in VR, providing a realistic scenario for train operations.

A study in [7] utilized augmented reality (AR) to simulate locomotive dashboard and controls for driver training in scenarios ranging from normal operation to emergency stop procedures. However, there could be certain limitations by using AR. For example, fixed physical markers placed on a surface are required to generate the virtual train cockpit which would require either a) a dedicated physical space for the cockpit or b) time to set-up the space for each training session. A full VR solution would have the entire train cockpit reproduced within the virtual environment, circumventing these issues. A user experience study on VR training simulators conducted in 2016 and 2018 [8] reported generally positive responses to the use of VR simulator and supplementing training in virtual environment. Nevertheless, users reported relatively poor quality of the simulation, due to the resolution and control method of VR simulator.

Given the rapid advancement in technology in recent years and state-of-the-art devices such as the HTC VIVE Pro Eye which has eye-tracking integrated into the VR headset, the realism and immersion of the simulation can be improved, making such implementations increasingly feasible.

\subsection{Human factors study in railway}

Human factors study in the railway industry consists of evaluation on technical skills and non-technical skills. The technical skills, lacking which can potentially cause maintenance errors and failures [9], can be evaluated by performance metrics, such as behavior, error rates, response speed, reaction time and completion time when the subject is performing tasks.

Non-Technical Skills (NTS) refer to the cognitive, social and personal resource skills that complement technical skills. Learning technical skills is the first main step, and improving NTS is the key to guaranteeing efficient and safe performance. Generally, the core NTS include teamwork, leadership, situation awareness, decision making, communication, coping with fatigue, stress management, etc. To identify rail industry's requirements for the incorporation of NTS into current Competency Management Systems, Madigan [10] conducted an interview, and the results indicated the importance of training people in the support roles, in order to maximize their ability to provide individualized support and to understand the link between technical and non-technical competency.

Andrew et al. [11] developed a three-phase approach to let the operational trainers understand how to integrate NTS into training, and the three phases are namely awareness phase, practice and feedback phase and reinforcement phase. In the awareness phase, the trainers are provided with a classroom-based introduction to the concept of NTS. The practice and feedback phase focuses on the development of realistic simulator scenarios that incorporate NTS into skills development, while the reinforcement phase focuses on collating trainers' feedback from their experiences of using and assessing NTS within training and simulation exercises. Results show that this method allows creation of a group of trainers who are able to develop and deliver NTS training materials.

Takashi [12] conducted interviews to collect the nontechnical error-prevention skills needed for railway drivers and designed specific situations to train these skills. For example, they found that drivers tend to apply old driving patterns to a new type of train, which could lead to potential mistakes. In order to prevent such mistakes, drivers were encouraged to increase the situation awareness by performing more checks and focus on the differences of the train types and environments.

Du et al. [13] evaluated four important NTS, which are situation awareness, decision-making, communication and self-management, in order to develop a NTS evaluation 
system for railway drivers. Principle component analysis (PCA) method and structural equation model (SEM) were then used in the model to assess the reliability and validity of the NTS scale. Nayak et al. [14] investigated the development of NTS for front line managers, considering the challenges for them to manage routine tasks and handle emergencies and abnormalities. The proposed generic NTS could complement their technical skill and enhance their overall managerial skills. In order to identify the most important NTS desired to improve safety of Indian Railway, Nayak et al. [15] identified 17 factors, including scientific working, recovery from error, emergency response, decision making, teamwork and so forth, through brainstorming and proposed a map of the complex relationships of these factors.

Dana et al. [16] reviewed over 150 papers about education in railway sector for both technical and non-technical skills and summarized 12 promising existing, new and emerging tools/methodologies, including e-learning, virtual/augmented reality, gaming environment and so forth.

In this paper, we describe a novel VR-based system that allows training and evaluation of stress and fatigue of railway operators that are the indicators of the most challenging tasks in training.

\section{System design and implementation}

\subsection{VR implementation}

The virtual scene for the training scenario consists of the surrounding environment, two engineering trains and a wagon. No digital data of any part are available, and all parts needed to be created from scratch.

The environment can be split into two parts, the workshop (Fig. 1a) and an area outside the workshop in the depot (Fig. $1 \mathrm{~b}$ and c), which are created to mimic the existing ones used by the SMRT Corporation (a multi modal transport operator in Singapore). All interactive parts are replicated with high precision based on pictures and manual measurements to guarantee that all parts match reality. The interactive parts in the environment are a manually operated point machine (Fig. 1c) that switches the direction of the rails and an aspect light that signals the permission to proceed on the rail, operated by the trainers.

The wagon consists of a lifting wagon with all its connectors required for coupling it to both engineering trains. No other functionality of the wagon is required, and all parts except the ones relevant for coupling are only a visual but not functional representation. The coupling portion is the same for both trains and wagon, which moves, collides, compresses and interlocks with each other, matching the

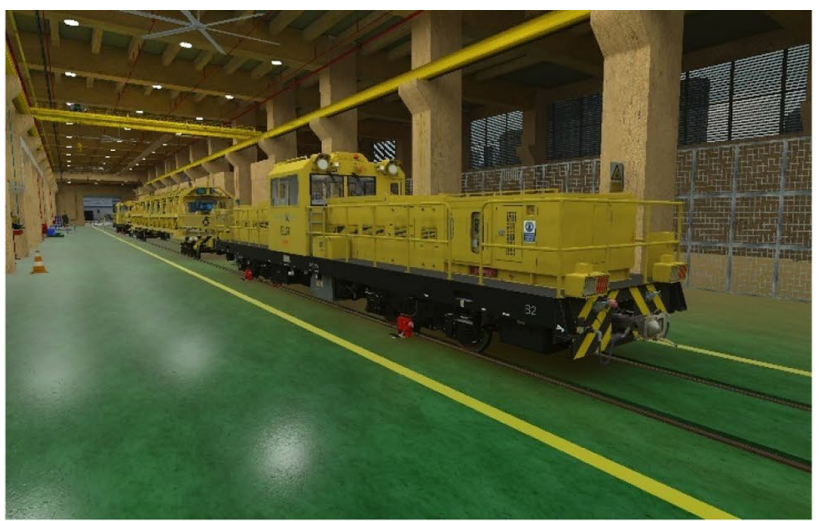

(a)

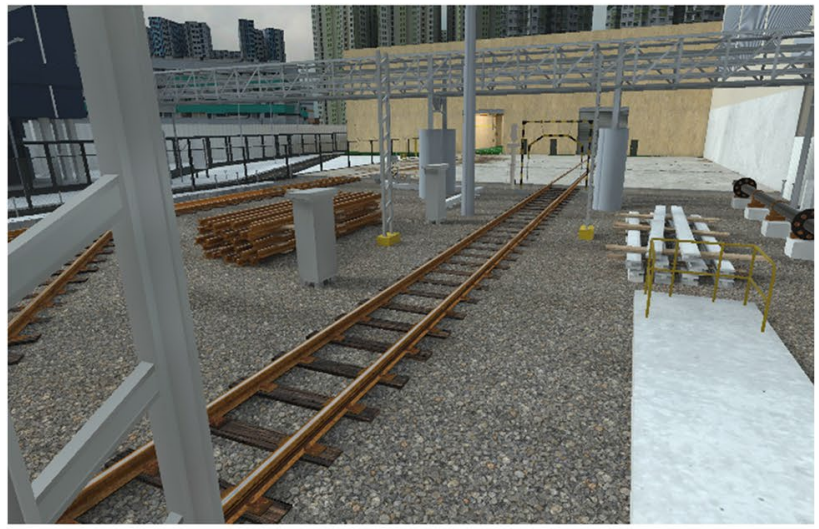

(b)

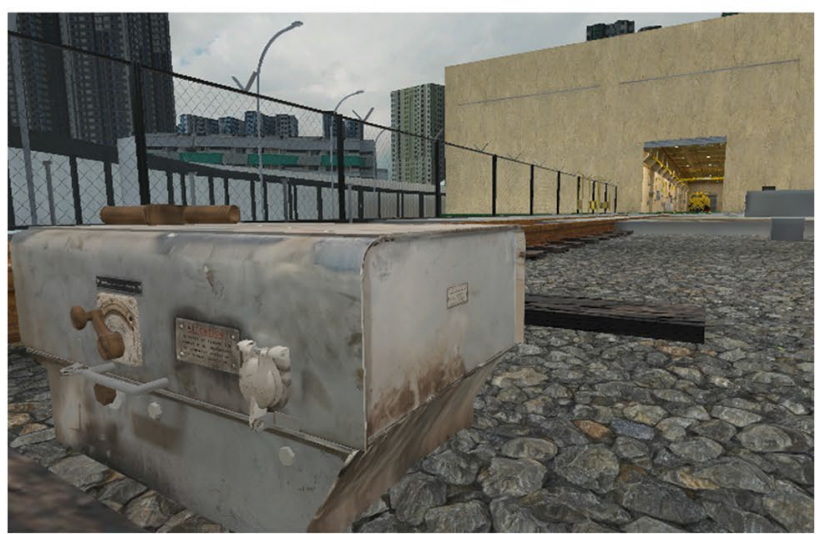

(c)

Fig. 1 An virtual environment for shunting training. a The workshop area. b A railway outside the workshop. c A point machine located beside the railway for switching the direction of the rail

real system. These details are needed in certain parts of the shunting workflow and will be observed by the trainees to conclude if a certain step of the coupling process was successful.

The engineering train was recreated with the focus on replicating all details and functionality as shown in Fig. 2. Every part required in the shunting operation was first measured and documented with pictures to allow a truthful 


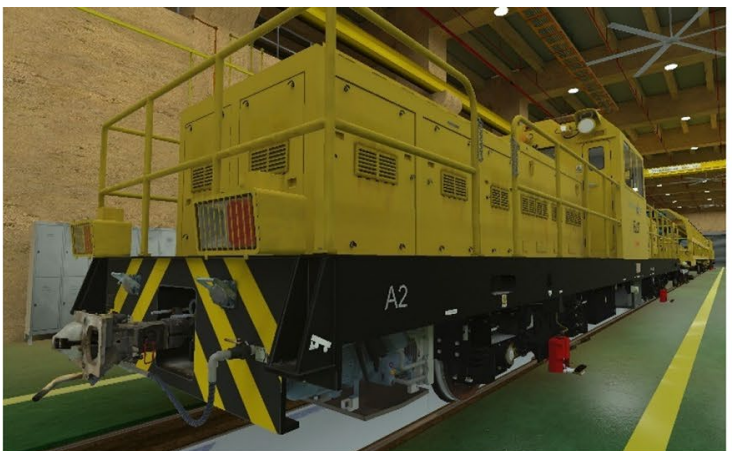

(a)

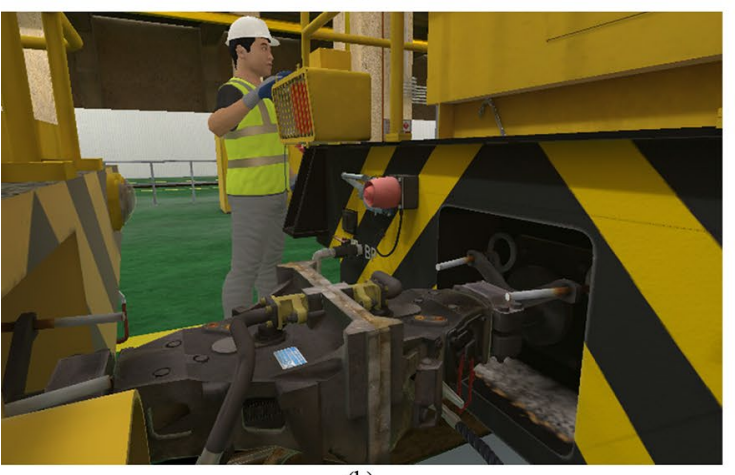

(b)

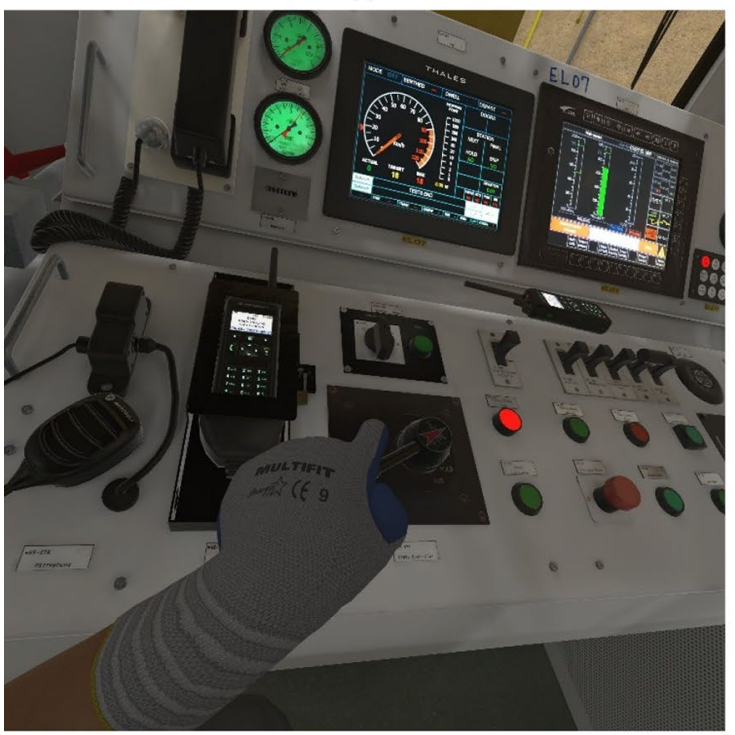

(c)

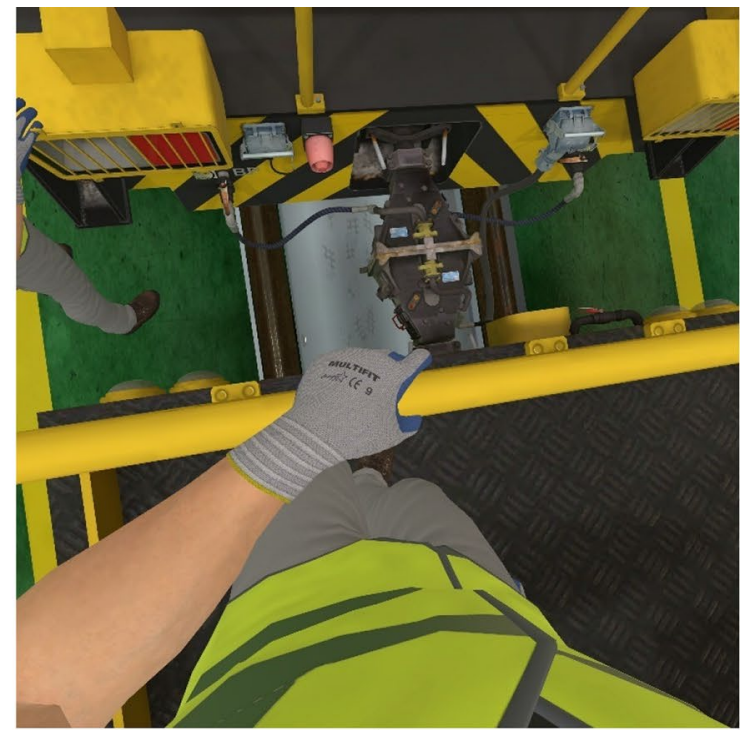

(d)

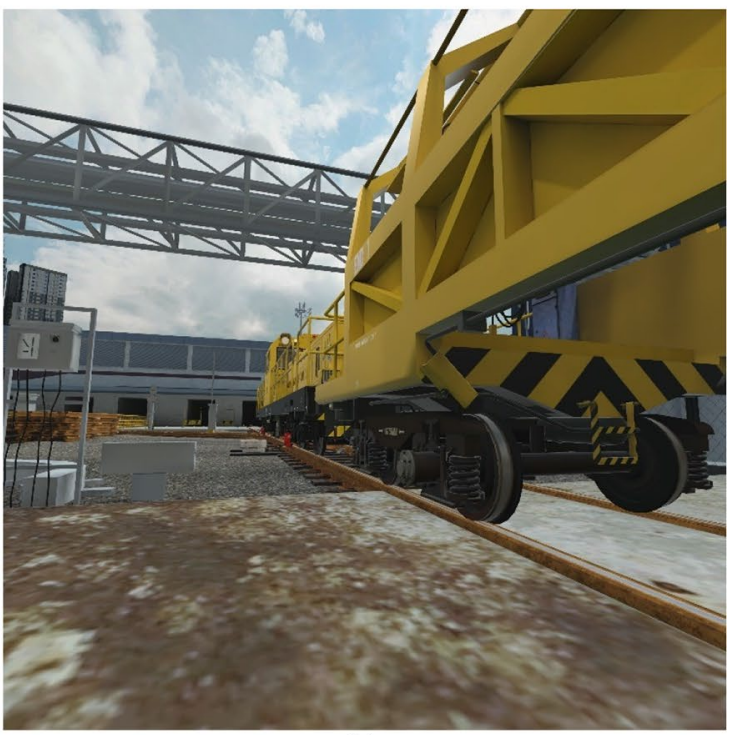

(e)

Fig. 2 An virtual engineering train. a The train inside the workshop. b An operator inspecting the train. $\mathbf{c}$ An operator grabbing the brake handle on the dashboard of the train. $\mathbf{d}$ The coupling/decoupling mechanism of the train $\mathbf{e}$ The train exiting the workshop

recreation of its virtual counterpart. The underlying system of invisible parts, such as the pressures in the pipes, has been analyzed, and a simulation system has been created that reacts to all input in the same way as would the real train for the required tasks. This includes the digital and analog systems in any part of the whole shunting procedure.

All interactive parts have been analyzed in their functionality and behavior, and for each part, the best implementation was chosen to mimic its behavior within the restrictions of virtuality, such as weightlessness or being unable to exert haptic force on the user. An example of a compromise required in VR is the insertion of a key into the keyhole. In the real world, this action is trivial, but in VR, moving a key into the keyhole without an opposing force as feedback upon collision turned out to be frustrating for the trainees. As a result, a method was 
implemented that will automatically rotate and align the key while it is moved into the hole. The same method was used for putting the brake handle or the handle of the point machine into their respective holes.

Dial switches use a system that takes into account not only the angular movement of the hand grabbing it but also the turn of the wrist. Early tests showed that there are two types of users, those who move the dial switch like they were using a wrench and those who turn it like they were using a screwdriver. The implemented system considers both.

Other haptic impressions were more easily mimicked in VR, such as the airflow of the aircon, by modulating the vibration motor in the controller. Flipping switches gives a short burst of vibration in the controller as feedback that the action has been performed. Haptic feedback also allows the trainee to control the train through feeling if the position of the master control handle is above or below neutral or if the brake controller is moved into a different mode, without the need to look at the controllers.

Good immersion also requires the surrounding sounds to match the visuals. Recordings have been made that capture specific sounds such as the starting of the aircon, a hammer hitting the wheels or ambient noise of the depot. For some tasks such as turning the aircon on or checking applied brakes, the sounds need to precisely mimic reality because they are the only way to confirm if the state of the train is correct. For testing the brakes, the trainee would hit the wheel with a hammer and listen for a ringing or dull sound, which would tell if the brakes are applied (dull sound) or not (ringing sound).

Other effects that improve the immersion but have no direct impact on the scenario have been added. This includes the simulation of echo and reverb in the workshop. The electric and air horn of the train will sound louder and with more echo in the workshop, compared to the open space. All sound effects have correct locations, and the trainee can properly hear the train approaching and the direction which the air horn signal comes.

Pre-recorded audio messages are used for radio messages to the trainees in the simulation. This happens if the trainee shunts past a red aspect, in which case the trainee will be asked over the radio to stop the train immediately since there was no permission given to shunt beyond the aspect light.

The system allows multiple trainees to collaboratively work in the same scenario. The collaborative aspect of the training system is powered by a server-client-based network architecture. Client PCs can join the server which does not need to be dedicated and normally runs in the background of an own client. The state of the whole virtual world is synchronized between all clients, including the full representation of all users and their avatars with eye-tracking data if an eye-tracking capable head mounted display (HMD) is used.
To achieve smooth positioning, the location and rotation are transferred relative to a dynamic origin. The origin can be the depot, wagon or trains. This is necessary so that clients will have precise control of the master control handle. All simulations happen on the server side, but the clients do smaller temporary simulations during interactions so that the user receives instantaneous feedback of their actions. Even in a local network, the delay of a pure server-side simulation was noticeable and made precise movements of the train hard, which lead to the implementation of the aforementioned approach.

The avatars use inverse kinematics to determine the virtual body of the trainee based on the data from the controllers and HMD. A virtual avatar is necessary for collaborative tasks in which hand gestures are used to communicate, such as signaling the remaining distance to the coupling or indicating to the operator to slow down or speed up. It is recommended to adjust the height of the avatar in the user interface to match the height of the trainee. If the avatar appears crouching or tiptoeing, it is an implication of height mismatch. The avatar will appear crouching if the trainee is shorter than the height set, or tiptoeing if the trainee is taller than the height set. It is also impossible for the avatar to reach further than its arm length so a taller person cannot fully use their reach of their arms if the avatar height is not properly set.

To achieve good graphical quality, lighting has been realistically replicated with a mixture of baked global illumination, light probes and real-time lighting. Most of the materials have albedo, normal, specular and occlusion maps based on pictures from the real train. This creates a natural impression since wear and tear from the real train is reproduced on the virtual train. Reflections use a mixture of reflection probes and real-time reflections, which are applied to the floor. These are achieved by rendering a view from a camera mirroring the position of the eyes at the ground level, which is used as reflection input. Annotated marks drawn on the train by operators are also included on the virtual train. Only defects or undesired states such as broken lights that would affect the training scenario had been artificially fixed for the virtual train.

To ensure that the training system is accurate, iterative feedback and testing had been done with the actual operators of SMRT until the point was reached where the operators did not find any unwanted difference in behavior between the real and virtual train. This feedback and testing loop also ensured that the end users have the chance to shape the interactive parts and behaviors of the application to their satisfaction and expectations.

The required hardware for the application is a steamVR compatible VR system and a powerful PC. Since the application uses skeletal-based hand tracking, the recommended controller would be the Valve Index Controller, but it also 
has been used and tested with conventional controllers, for which a default grip position of the hand is assumed. The minimum recommended hardware requirements for the PC are a GTX 1080 graphic card, 16 GB of RAM and an i7-6800 k CPU.

\subsection{System interface for stress and fatigue monitoring}

The system provides an interface for the trainers to supervise the trainees in the virtual environment, which displays the current states of the train such as power status, wheel chock status, brake pressure, etc. The interface overlays the VR scene at the upper left corner and is visible only to the trainers. This enables the trainers to quickly help a trainee if they are stuck in a procedure. It can further show the live stress and fatigue values of the trainee (Fig. 3), and they can be gathered on a by-task basis to visualize trends and enable day-to-day performance comparison. The fatigue and stress recognition algorithms are implemented based on the stateof-art methods published in [17-20], respectively.

In the real-time stress and fatigue monitoring as shown in Fig. 3, the green and red ribbon represent the levels of fatigue and stress, where green color indicates a low level of fatigue/stress, red indicates a high level of fatigue/stress, and a yellow color indicates a medium level of fatigue/stress. The system supports two assessment modes. In the first mode, the trainers have to click the tags in the task panel to indicate which task is being executed at the current moment (as illustrated in Fig. 3, Task 5.4 is selected by the trainer as it is being executed and monitored). As a result, the average stress and fatigue during each task performance is displayed upon the completion of that task as shown in Fig. 3.

A comprehensive report is generated after the completion of tasks, which includes mean fatigue/stress states of every 5-min interval and the mean fatigue/stress states over every selected task. A result analytic is also included in the report, which lists a summary of the intervals of low/medium/high stress/fatigue, tasks of low/medium/high stress/fatigue, average fatigue/stress over the entire session, percentage of low/ medium/high stress/fatigue intervals over the entire durations and percentage of low/medium/high stress/fatigue tasks over all tasks.

In the second mode, if the trainers are busy monitoring multiple trainees and do not have time to click the task tags (Fig. 4), the system provides assessment report of mean fatigue/stress states of every 5-min interval. In the report, mean fatigue/stress states of every 5-min interval, a summary of the intervals of low/medium/high stress/fatigue, average fatigue/stress over the entire session and percentage of low/medium/high stress/fatigue intervals over the entire durations are presented.

Further convenience options are available to adjust the interface or skip certain procedures and events that happen in a training session are logged and available as a text file. Every training session requires both a session name and trainee name.

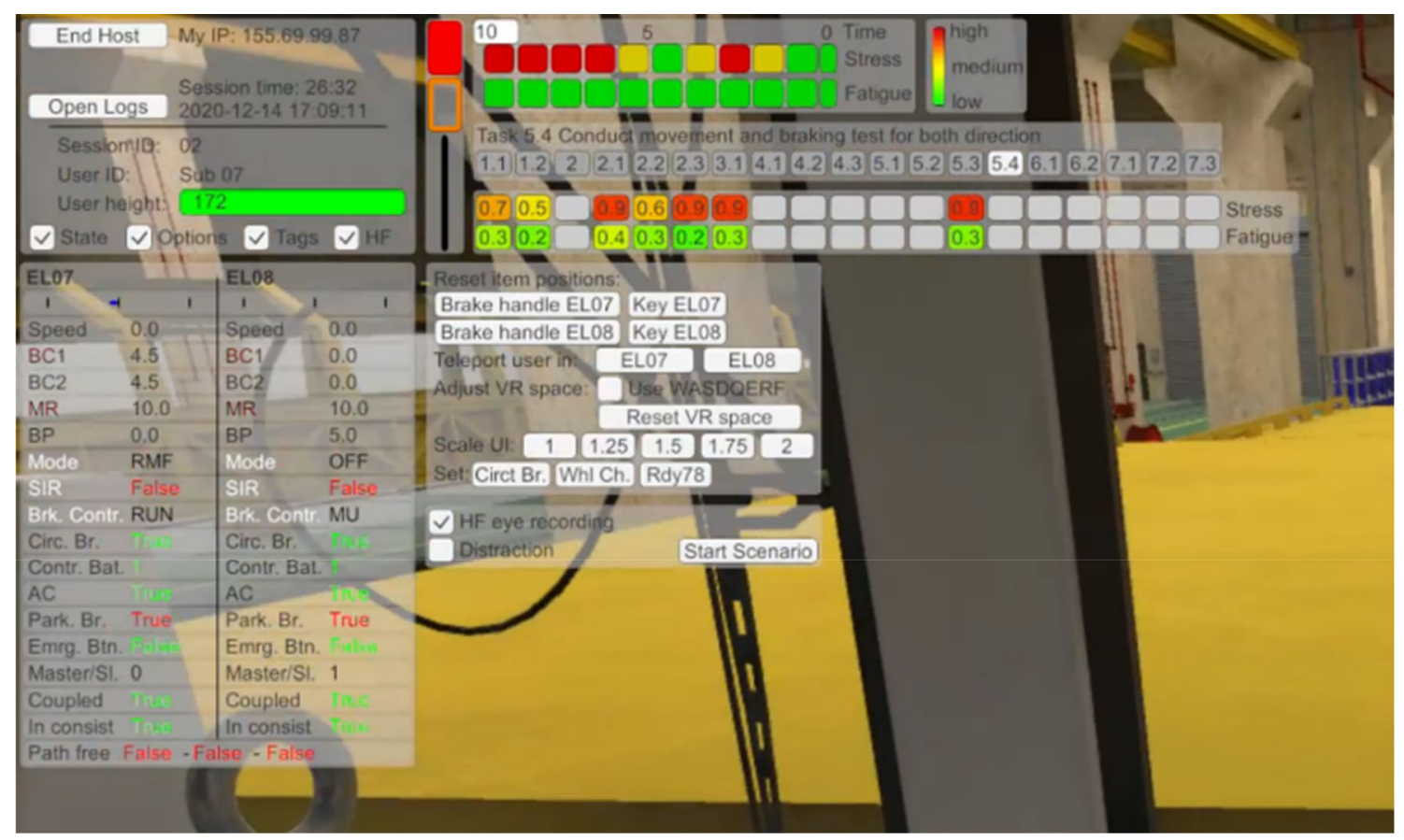

Fig. 3 Interfaces when trainers click task tags 


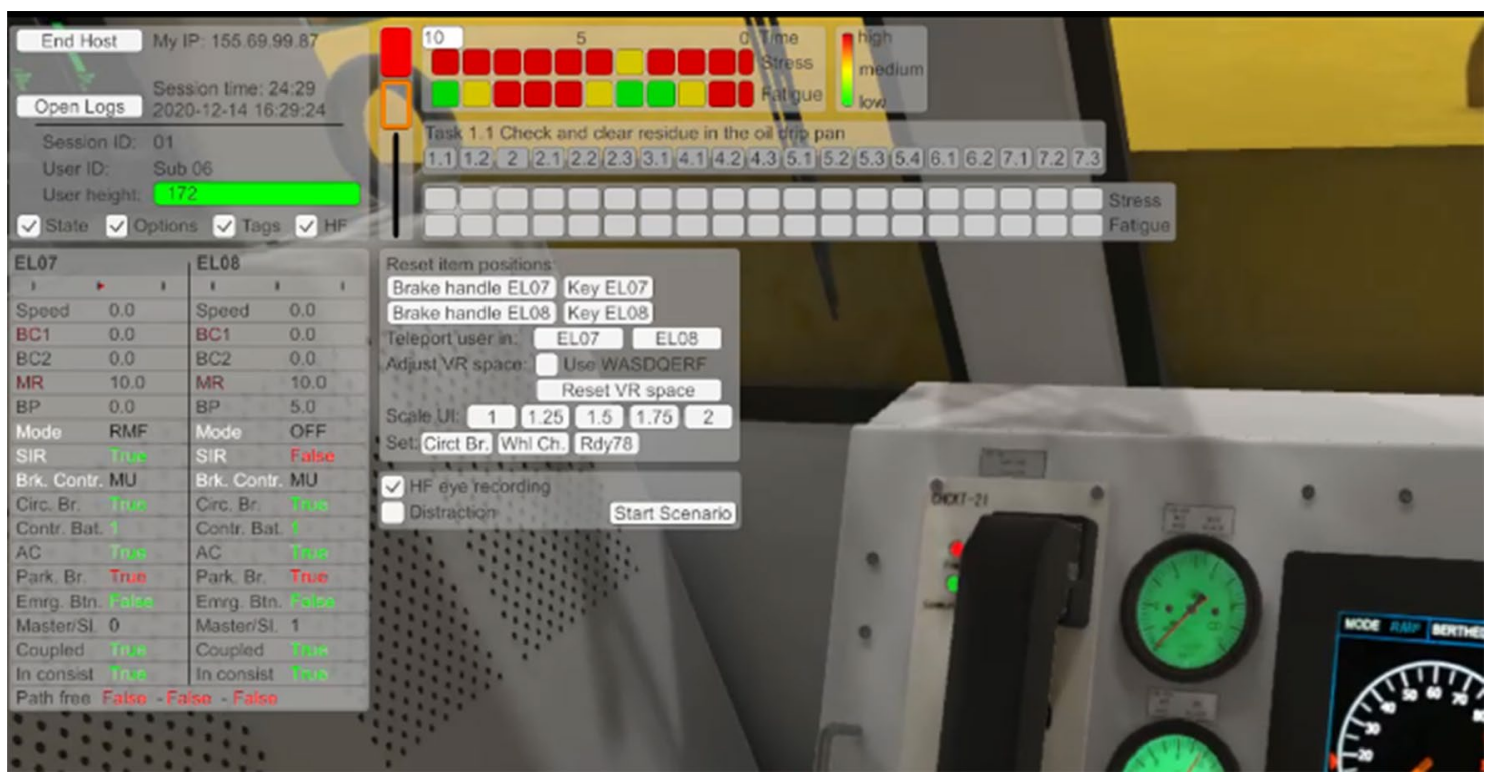

Fig. 4 Interfaces when trainers do not click task tags

An optional feature for the trainee is a virtual touch tablet that contains information defined by the supervisor. This would normally be the description of all tasks and, if made available to the trainee, can help trainees to solve problems by themselves instead of relying on the supervisor. The tablet is created dynamically and appears if a certain text file is in the folder of the application. The text in the file will then be visible on the tablet, supporting common text formatting methods. The trainee can interact with it, like with any real world tablet. It is not synchronized over the network so that trainees can have different content on their tablets.

\section{Experiment}

\subsection{Subjects}

Twelve technically skilled trainee subjects and 3 trainer subjects participated in the experiment. Among the subjects, 8 are male, and the age of subjects ranges from 23-59-yearsold. All subjects were recruited from SMRT Train Ltd. Specifically, the trainee subjects are competent train operators that have undergone rigorous on-site training before they assumed duty at SMRT.

\subsection{Scenario description}

The subjects were asked to conduct shunting operation scenario in VR. In the designed scenario, the signal aspect is red by default and can be switched to yellow by the trainers. The master Executive Train Operator (ETO) is expected to respond and stop shortly before the red aspect and wait for it to be changed to yellow. If the master ETO passes the red aspect, Operations Control Center (OCC) would inform ETO to stop immediately. After the trainer changes the aspect to yellow, ETO should respond and proceed. ETO also needs to sound the horn to alert others of the approaching train. The speed should be controlled at all times (should not exceed $5 \mathrm{~km} / \mathrm{h}$ in the workshop and $18 \mathrm{~km} / \mathrm{h}$ in the depot and pit). If the speed exceeds $18 \mathrm{~km} / \mathrm{h}$, the consist will stall, and the ETO has to restart the consist. The corresponding timeline is illustrated in Fig. 5.

\subsection{Experiment procedure}

Multi-source data, including eye-tracking, behavioral and logs from VR were collected during the experiment. Two sets of questionnaires were designed and distributed to the trainers and trainees subjects. For trainers, the questionnaires are used for trainee performance evaluation and feedback toward the VR simulation. For trainees, the questionnaires are mainly for feedback toward the VR training. During the experiment, the trainers were in the same room with the trainees, who were going through the VR scenarios. Three researchers attended the experiment to help with the VR setup and acted as the slave ETO/ground shunter. The experimental procedure is shown in Table 1.

\subsection{Device}

In the experiment, the HTC VIVE Pro Eye VR headset with integrated eye-tracking technology is used in the implemented VR training system. 


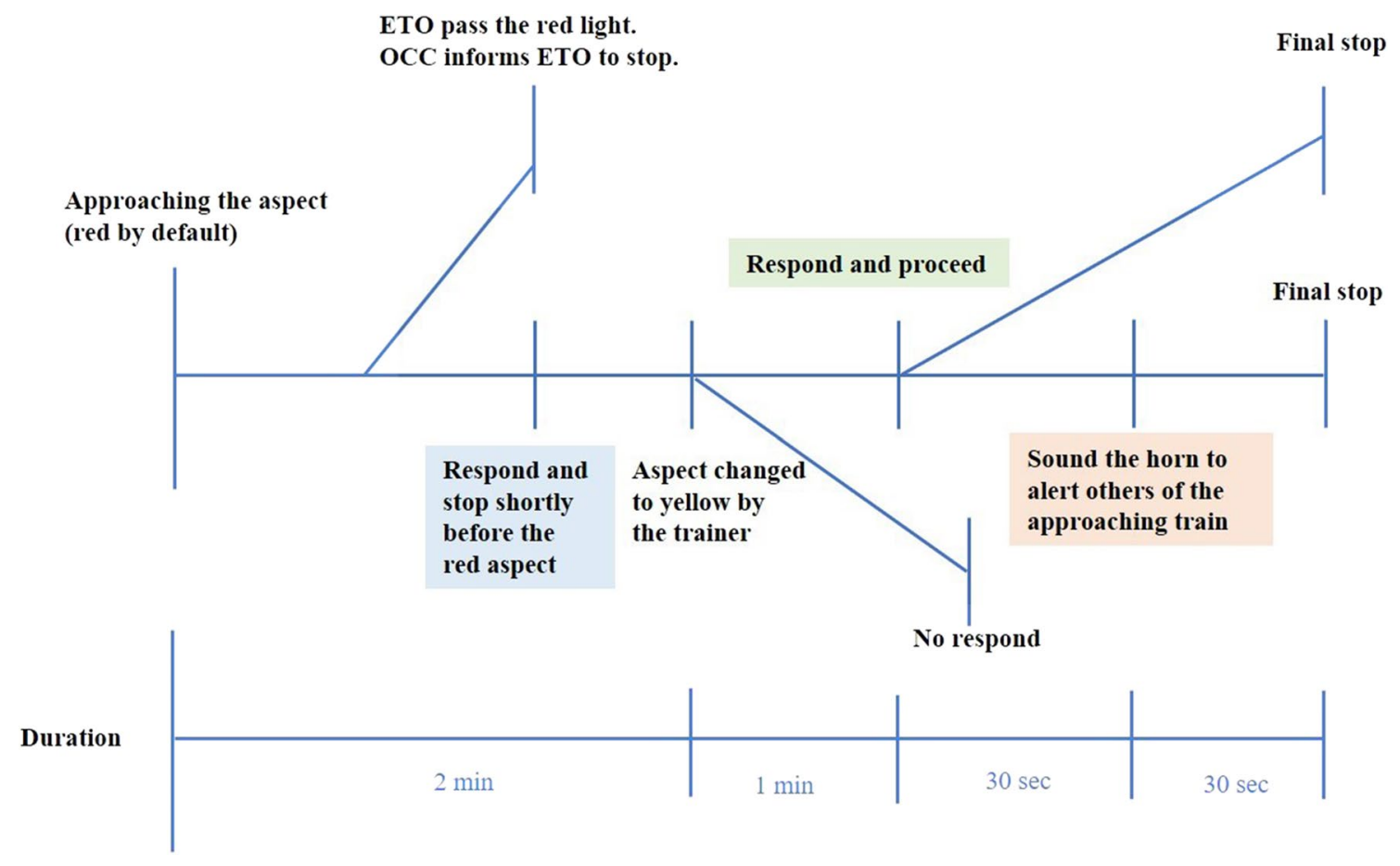

Fig. 5 Timeline of scenario (duration not drawn to scale)

\subsection{Questionnaires}

Trainees completed intake and post-experiment questionnaires before and after the experiment, respectively. The intake questionnaire for trainees include personal information such as gender, age, experience, working position and perceived mental states such as their fatigue/stress level on that day. Figure 6 shows the specific items that were used to understand fatigue and stress states in the questionnaires.

The post-experiment questionnaire includes three partsquestions on the fatigue/stress states, feedback about VR experience and evaluation of the VR-based training simulator. The VR experience questions were modified based on the study in [21]. Eleven questions are chosen to assess the feeling of presence, reality, stressfulness, tiredness, effectiveness, attention to scenes and sound within VR. To compare the VR-based training simulator with other traditional training methods, such as video training, lecture training and on-site training, 8 questions are proposed. In addition, trainees are required to tick their perceived advantages and disadvantages of VR-based training, as shown in Fig. 7.

The trainers received three questionnaires including intake, trainee performance assessment questionnaire and post-experiment questionnaires. The intake questionnaire records the information of experiment time, trainer ID and experience. The trainee performance assessment questionnaires are used by the trainers to report the performance of the trainee after observing his/her behaviors and activities in VR. The post-experiment questionnaire of trainers includes feedback about the effectiveness of training in VR and on-site, the difficulty of tasks in VR and real life and the possibility of using VR for SMRT shunting training. Questions comparing the VR training simulator with other traditional training methods, such as video training, lecture training and on-site training were also asked. In addition, trainers were required to tick their perceived advantages and disadvantages of VR-based training.

Hypothesis The data collected from the experiment are used to validate three hypotheses as listed below.

- Hypothesis 1: the VR-based training will not induce extra fatigue/stress and physical uncomfortableness to the trainees.

- Hypothesis 2: The VR-based simulation provides a suitable environment for shunting operation training.

- Hypothesis 3: Trainers and trainees prefer to utilize VR-based simulation for shunting operation training than other traditional training methods, such as video training, lecture training and on-site training. 


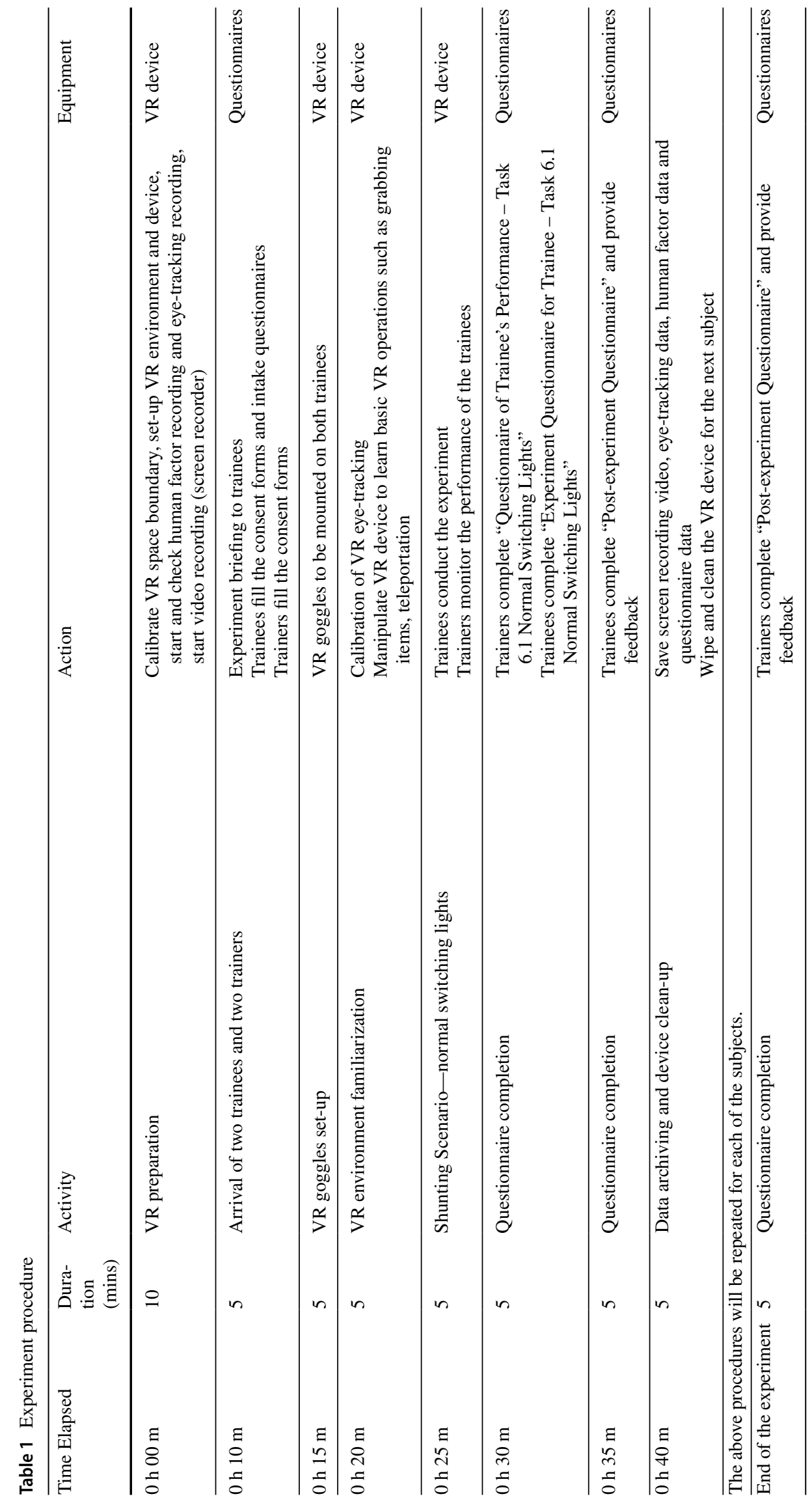




\begin{tabular}{|c|c|c|c|c|c|c|}
\hline \multicolumn{7}{|l|}{ How do you feel? } \\
\hline Feeling active & 1 & 2 & 3 & 4 & 5 & Completely exhausted \\
\hline Not at all stressful & 1 & 2 & 3 & 4 & 5 & Extremely stressful \\
\hline \multicolumn{7}{|c|}{ Additional Questions: } \\
\hline \multicolumn{7}{|c|}{ 1) How tired are your eyes? } \\
\hline 1 & & 2 & & & 3 & 4 \\
\hline \multicolumn{7}{|c|}{ 2) How clear is your vision? } \\
\hline 1 & & 2 & & & 3 & 4 \\
\hline \multicolumn{7}{|c|}{ 3) How do your eyes feel? } \\
\hline 1 & & 2 & & & 3 & 4 \\
\hline \multicolumn{7}{|c|}{ 4) How tired is your back? } \\
\hline 1 & & 2 & & & 3 & 4 \\
\hline \multicolumn{7}{|c|}{ 5) How tired is your neck? } \\
\hline 1 & & 2 & & & 3 & 4 \\
\hline \multicolumn{7}{|c|}{ 6) How severe is your headache? } \\
\hline 1 & & 2 & & & 3 & 4 \\
\hline \multicolumn{7}{|c|}{ 7) How sleepy do you feel? } \\
\hline 1 & & 2 & & & 3 & 4 \\
\hline
\end{tabular}

Fig.6 Questionnaire on the mental states of trainees

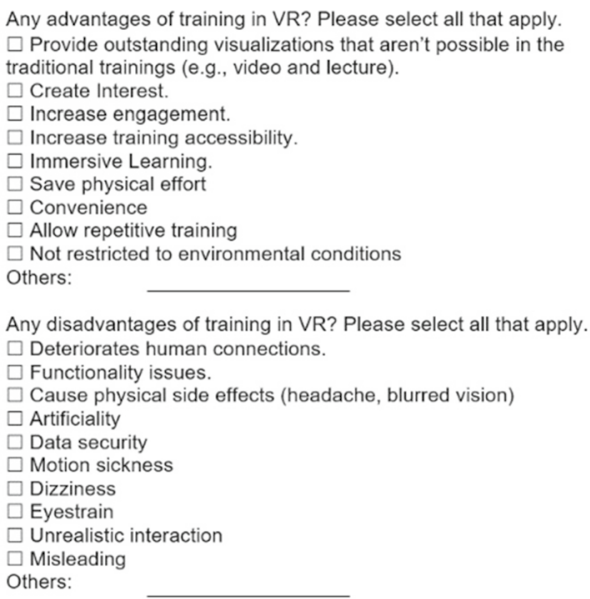

Fig. 7 Questionnaire on advantages and disadvantages of training in VR

\section{Results and discussion}

The data from the questionnaires given to trainees and trainers were analyzed, and the results are shown in the following sections.

\subsection{Trainees' physical and mental states before and after experiments}

Table 2 shows the changes of both physical and mental states of trainees before and after the experiment. It was found that after the experiments, the average changes across all 12 subjects in all these items are minor. T-test was conducted to compare the mean value between
Table 2 Physical and mental states change before and after experiment

\begin{tabular}{lll}
\hline Items & $\begin{array}{l}\text { Changes between post- } \\
\text { and pre-experiment }\end{array}$ & P value of t-test \\
\hline Overall fatigue & 0 & 0.75 \\
Overall stress & 0.25 & 0.43 \\
Eye tiredness & 0.25 & 0.34 \\
Vision (visual acuity) & 0 & 0.37 \\
Strain eye & -0.25 & 0.08 \\
Back pain & -0.125 & 0.17 \\
Neck pain & 0.375 & 0.44 \\
Headache & -0.125 & 0.59 \\
Sleepiness & -0.375 & 0.08 \\
\hline
\end{tabular}

Table 3 Trainees' post-experiment feedbacks

\begin{tabular}{ll}
\hline Subjective feelings & Average \\
\hline 1. presence of VR experience & 3.33 \\
2. realism of VR experience & 3.50 \\
3. stress of shunting in VR & 2.75 \\
4. sense of movement in VR & 3.92 \\
5. fatigue after VR experience & 2.25 \\
6. attention to aspect in VR & 4.33 \\
7. how real the sound is in VR & 3.67 \\
8. attention to ambient sound & 2.92 \\
9. effectiveness of the shunting training in VR & 4.25 \\
10. effectiveness of the on-site training & 4.36 \\
11. difficulty of task in VR comparing to real life & 2.67 \\
12. preference to use VR over video & 4.00 \\
13. preference to use VR over lecture & 4.00 \\
14. preference to use VR over on-site training & 2.67 \\
15. VR learn faster than videos & 4.50 \\
16. VR learn faster than lectures & 4.50 \\
17. level of trust in VR & 3.50 \\
\hline
\end{tabular}

post- and pre-experiment. The overall fatigue levels and visual acuity remain unchanged. The overall stress, eye tiredness and neck pain increased a little. Other states, such as eye strain, back pain, headache and sleepiness decreased. In general, the results of t-test in all the states are above 0.05 . Accordingly, hypothesis 1 is confirmed.

\subsection{Trainees' feedback on the VR training simulator}

By averaging the ratings from the post-experiment survey questionnaires across all trainees, the following results are shown in Table 3. The average rating of presence is 3.33/5, which indicates that during the VR training, the trainees had a strong sense of being present in the virtual world. The average rating of realism is $3.5 / 5$, which indicates that 
the trainees felt shunting operation in VR were real to them almost all the time. The trainees also reported that they can sense the movement during training (3.92/5), which proves again that the VR scenario created a realistic environment. In addition, the trainees rated that they paid a lot of attention to visuals-such as the aspect in VR (4.33/5), though less attention to audios-such as the ambient sound (2.92/5). However, they still rated that the sound in the VR scenarios was real to them with an average rating of 3.67/5.

The stress and fatigue level after the VR training were lower than 3, which indicates that the VR training did not bring any extra stress and fatigue to the trainees. The effectiveness of the training in VR was rated as 4.25/5, which is slightly lower than the effectiveness of the on-site training (4.36/5), and the preference is still with on-site training. However, the trainees rated that they preferred to use VR over other traditional training methods such as video- and lecture-based ones. Our finding of preference for VR training over its traditional counterparts is in agreement with that reported in [7], which also shows positive feedback for new VR training from the majority of users. The trust toward VR training is also high (3.50/5), which could be a sign that VR is suitable for shunting training. Thus, hypothesis 2 is confirmed, and hypothesis 3 is partially confirmed by the trainees.

\subsection{Trainers' feedback on the VR training simulator}

Questionnaires have been given to the trainers to get feedback from them about training in VR as well. As shown in Table 4, the trainers agreed that training in VR is effective, and they preferred to use it over videos and lectures, though on-site training is still considered as the best training method. They also mentioned that the possibility of using VR for shutting training is very high, as the VR system can help individuals learn the shunting operation faster than training based on videos and lectures. Thus, hypothesis 2

Table 4 Trainers' post-experiment feedbacks

\begin{tabular}{ll}
\hline Post-experiment feedbacks & Average \\
\hline 1. effectiveness of the shunting training in VR & 4 \\
2. effectiveness of the on-site training & 4.7 \\
3. difficulty of task in VR comparing to real life & 3 \\
4. the possibility of using VR for SMRT shunting training & 4 \\
5. preference to use VR over video & 4 \\
6. preference to use VR over lecture & 4 \\
7. VR training systems can help individuals learn the shunt- & 4 \\
ing operation faster than training by videos methods & \\
8. VR training systems can help individuals learn the shunt- & 4.2 \\
ing operation faster than training by lectures & \\
\hline
\end{tabular}

is confirmed, and hypothesis 3 is partially confirmed by the trainers.

\subsection{Feedback on the advantages and disadvantages of training in VR}

Several possible advantages and disadvantages were listed in the questionnaire based on literature review about the VR-based training. Both trainers and trainees were asked to indicate based on their perception toward VR-based training. The percentage of trainers/trainees who agreed on each advantage/disadvantage point is shown in Tables 5 and 6 , respectively. Compared with trainers, trainees tended to tick more advantage points of the VR training. Four listed advantages, namely "increase engagement", "increase training accessibility", "immersive learning", "allow repetitive training" were selected by all trainees. Whereas only two advantages of the VR training, namely "save physical effort" and "allow repetitive training" were selected by all trainers. For disadvantage of the VR-based training, the focuses are

Table 5 Feedback on advantages of training in VR

\begin{tabular}{|c|c|c|}
\hline Advantages of training in VR & $\begin{array}{l}\text { Trainees' } \\
\text { feedback }\end{array}$ & $\begin{array}{l}\text { Trainers' } \\
\text { feedback }\end{array}$ \\
\hline $\begin{array}{l}\text { Provide outstanding visualizations that aren't } \\
\text { possible in the traditional trainings }\end{array}$ & $83 \%$ & $50 \%$ \\
\hline Create interest & $83 \%$ & $33 \%$ \\
\hline Increase engagement & $100 \%$ & $50 \%$ \\
\hline Increase training accessibility & $100 \%$ & $67 \%$ \\
\hline Immersive learning & $100 \%$ & $67 \%$ \\
\hline Save physical effort & $75 \%$ & $100 \%$ \\
\hline Convenience & $83 \%$ & $17 \%$ \\
\hline Allow repetitive training & $100 \%$ & $100 \%$ \\
\hline Not restricted to environmental conditions & $83 \%$ & $33 \%$ \\
\hline
\end{tabular}

Table 6 Feedback on disadvantages of training in VR

\begin{tabular}{lll}
\hline Disadvantages of training in VR & $\begin{array}{l}\text { Trainees' feed- } \\
\text { back }\end{array}$ & $\begin{array}{l}\text { Trainers' } \\
\text { feedback }\end{array}$ \\
\hline Deteriorates human connections & $17 \%$ & $17 \%$ \\
Functionality issues & $25 \%$ & $5 \%$ \\
Cause physical side effects (headache, & $42 \%$ & $67 \%$ \\
$\quad$ blurred vision) & & \\
Artificiality & $25 \%$ & $33 \%$ \\
Data security & $8 \%$ & 0 \\
Motion sickness & $58 \%$ & $100 \%$ \\
Dizziness & $25 \%$ & $67 \%$ \\
Eye strain & $42 \%$ & $67 \%$ \\
Unrealistic interaction & $33 \%$ & $5 \%$ \\
Misleading & 0 & 0
\end{tabular}


mainly on physical side effects (headache, blurred vision), motion sickness and eye strain. However, very few trainees reported discomforts during or after the experiment despite indicating it in the questionnaires. Besides the advantages and disadvantages listed in the questionnaires, the trainers and trainees were also asked to write down any comment regarding the training in VR. We received feedback from some trainees that VR did help in better memorization of the procedure, but cannot fully mimic the feel of the physical objects. In addition, physical touch, the weight of items, physical movement up and down of the real locomotive are absent from VR, and no effort to move is needed due to teleportation which further detracts from reality.

\section{Conclusion}

In this paper, we present a VR-based training for shunting operations in railway. An experiment was carried out to validate the implemented VR-based training. Twelve trainees and three trainers were recruited in the experiment. Questionnaires were distributed to both trainees and trainers to get their feedback toward the VR-based training. The obtained results confirmed the hypothesis that the VR-based training will not induce extra fatigue/stress and physical uncomfortableness to the trainees; the VR-based simulator provides a suitable virtual alternative for shunting operation training and both trainees and trainers reported a positive VR experience. Furthermore, trainers and trainees prefer to use a VR-based simulator for shunting operation training than other traditional training methods, such as video training and lecture training.

The VR-based training comes with the unique human factors evaluation which could monitor the fatigue/stress level of the user in real-time and generate a comprehensive report after the training regarding mean fatigue/stress states of every 5-min interval and the mean fatigue/stress states over every selected task, etc. The report also include analytics results such as a summary of the intervals of low/medium/ high stress/fatigue, tasks of low/medium/high stress/fatigue, average fatigue/stress over the entire session, percentage of low/medium/high stress/fatigue intervals over the entire durations and percentage of low/medium/high stress/fatigue tasks over all tasks.

In the next step, we are going to analyse the biosignal and behavioral data collected from the experiment and assess the competence of the trainees.

Acknowledgements This research is supported by the National Research Foundation, Singapore, under its International Research Centers in Singapore Funding Initiative. Any opinions, findings and conclusions or recommendations expressed in this material are those of the author(s) and do not reflect the views of National Research Foundation, Singapore.

Funding Open Access funding enabled and organized by Projekt DEAL.

\section{Declarations}

Conflict of interest The authors declare that there is no conflict of interest.

Open Access This article is licensed under a Creative Commons Attribution 4.0 International License, which permits use, sharing, adaptation, distribution and reproduction in any medium or format, as long as you give appropriate credit to the original author(s) and the source, provide a link to the Creative Commons licence, and indicate if changes were made. The images or other third party material in this article are included in the article's Creative Commons licence, unless indicated otherwise in a credit line to the material. If material is not included in the article's Creative Commons licence and your intended use is not permitted by statutory regulation or exceeds the permitted use, you will need to obtain permission directly from the copyright holder. To view a copy of this licence, visit http://creativecommons.org/licenses/by/4.0/.

\section{References}

1. Truong, K.P., van Leeuwen, D.A.: Automatic discrimination between laughter and speech. Speech Commun. 49(2), 144-158 (2007)

2. Li, F., Chen, C.-H., Xu, G., Chang, D., Khoo, L.P.: Causal factors and symptoms of task-related human fatigue in vessel traffic service: A task-driven approach. The Journal of Navigation 73(6), 1340-1357 (2020)

3. Xu, J., et al.: A VR-based the emergency rescue training system of railway accident. Entertainment Computing 27, 23-31 (2018)

4. Nwaeke, L.I., Obiekwe, O.: Impact of manpower training and development on organizational productivity and performance: A theoretical review. European Journal of Business and Management 9(4), 153-159 (2017)

5. Gegenfurtner, A., Zitt, A., Ebner, C.: Evaluating webinar-based training: a mixed methods study of trainee reactions toward digital web conferencing. Int. J. Train. Dev. 24(1), 5-21 (2020)

6. Guan, C., Chang, L., and Xu, H.: "The simulation of traction and braking performance for high-speed railway virtual reality system," in 2013 5th International Conference and Computational Intelligence and Communication Networks, pp. 631-634: IEEE (2013)

7. Ćwil, M., and Bartnik, W.: "Physically extended virtual reality (PEVR) as a new concept in railway driver training," in International Conference on Human-Computer Interaction, 2019, pp. 230-242: Springer.

8. Papa, S., Lanzotti, A., Di Gironimo, G., Balsamo, A., Ideas, F.: A new interactive railway virtual simulator for testing preventive safety. Computers in Railways XVI: Railway Engineering Design and Operation 181, 367 (2018)

9. James, A. T., Gandhi, O. P., and Deshmukh, S. G.: "Assessment of failures in automobiles due to maintenance errors," International Journal of System Assurance Engineering and Management, vol. 8, no. 4, pp. 719-739, 2017/12/01 2017.

10. Madigan, R., Golightly, D., Madders, R., Sharples, S., Shorrock, S., and Waterson, P.: "Rail industry requirements around non-technical skills," in Contemporary Ergonomics and Human Factors, 
2015. International Conference on Ergonomics and Human Factors, CRC Press, Taylor and Francis Group, Daventry, Northamptonshire, 2015.

11. RUSSELL, A. J.: "A Model for the Development of Railway Trainers in Integrating Non-Technical Skills into Training and Assessment: An International Case Study of Train Driver Trainer Skills Development."

12. Enomoto, T. (2011). A study of extracting and sharing non-technical skills among train drivers in JR east.

13. Du, Y., Fang, W., and Niu, K.: "A Method for Evaluation System of Railway Driver Non-Technical Skills Construction," in 2018 International Conference on Intelligent Rail Transportation (ICIRT), 2018, pp. 1-5: IEEE.

14. Nayak, S., Tripathy, S., Dash, A.: Non-technical skill development strategy to enhance safety performance of railway system: an interpretive structural modelling approach. Int. J. Bus. Excell. 19(2), 168-188 (2019)

15. Nayak, S., Tripathy, S., Dash, A.: Role of non technical skill in human factor engineering: a crucial safety issue in Indian Railway. International Journal of System Assurance Engineering and Management 9(5), 1120-1136 (2018)

16. Sitányiová, D., and Mašek, J.: "New Training Schemes for The Future Education in Railway Sector," in MATEC Web of Conferences, 2018, vol. 235, p. 00014: EDP Sciences.

17. Giannakakis, G., Grigoriadis, D., Giannakaki, K., Simantiraki, O., Roniotis, A., and Tsiknakis, M.: "Review on psychological stress detection using biosignals," IEEE Transactions on Affective Computing, 2019.

18. Herten, N., Otto, T., Wolf, O.T.: The role of eye fixation in memory enhancement under stress-An eye tracking study. Neurobiol. Learn. Mem. 140, 134-144 (2017)

19. Armougum, A., Gaston-Bellegarde, A., Joie-La Marle, C., and Piolino, P., "Physiological investigation of cognitive load in reallife train travelers during information processing," Applied Ergonomics, vol. 89, p. 103180, 2020.

20. Hirt, C., Eckard, M., and Kunz, A.: "Stress generation and nonintrusive measurement in virtual environments using eye tracking," Journal of Ambient Intelligence and Humanized Computing, pp. 1-13, 2020.

21. Seinfeld, S., et al.: Influence of music on anxiety induced by fear of heights in virtual reality. Front. Psychol. 6, 1969 (2016)

Publisher's Note Springer Nature remains neutral with regard to jurisdictional claims in published maps and institutional affiliations.

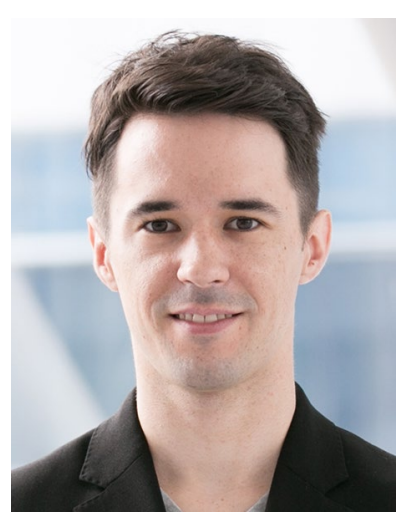

Benedikt Tschoerner is specialized in developing virtual reality applications for the industry and works in this field since graduating in 2015 with a Master in Visual Computing from the University of Koblenz-Landau. His research is mainly focused on the integration of multidisciplinary aspects into virtual reality.

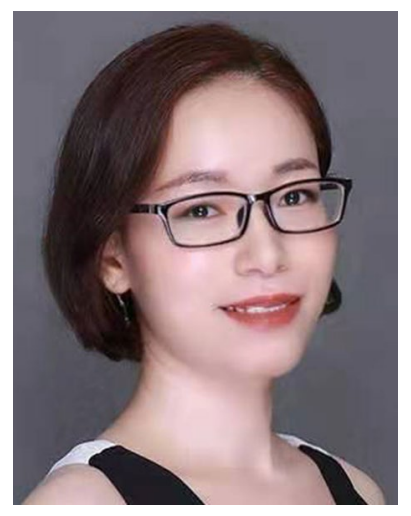

Fan Li received the B.Sc. degree from the School of Aeronautic Science and Engineering, Beihang University, China, in 2013. She received her M.Sc. and Ph.D. degrees in Mechanical Engineering from the School of Mechanical and Aerospace Engineering, Nanyang Technological University, Singapore, in 2015 and 2020. She is currently a Research Fellow of Fraunhofer Singapore. Her research interests lie in human factors, safety management, and machine learning.

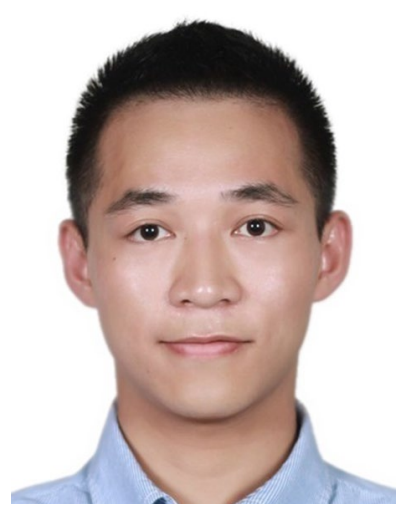

Zirui Lan received his B.Sc. in Computer Science and Technology from Sun Yat-Sen University, China, and joint Ph.D. from Nanyang Technological University, Singapore and Graz University of Technology, Austria. He is currently with Fraunhofer Singapore. His research interests include machine learning and its application.

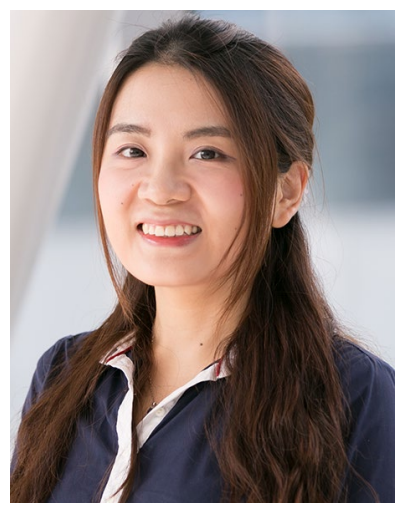

Yisi Liu is a Principal Research Fellow at Fraunhofer Singapore. She received her B.Eng. in Information Security from University of Science and Technology Beijing, M.Sc. and Ph.D. degrees in Electrical Engineering from Nanyang Technological University. Her research interests include human factors study, signal processing, and artificial intelligence.

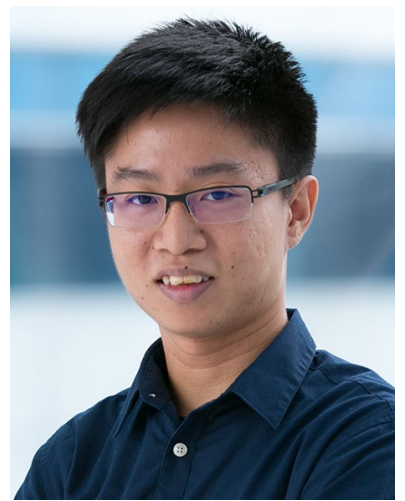

Wei Lun Lim received his B.Eng. and Ph.D. degrees in Electrical and Electronic Engineering from Nanyang Technological University, Singapore. He is currently a Research Fellow at Fraunhofer Singapore with research interests in topics involving machine learning for human factors. 


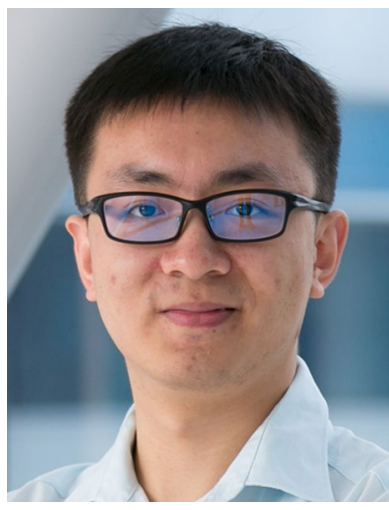

Jian Cui is now working as a Research Fellow at Fraunhofer Singapore, Nanyang Technology in Mathematics from Dalian University of Technology and Ph.D. in School of Computer Science and Engineering from Nanyang Technological University. His research areas include machine learning, deep learning, electroencephalogram (EEG) signals processing, and virtual interaction techniques.
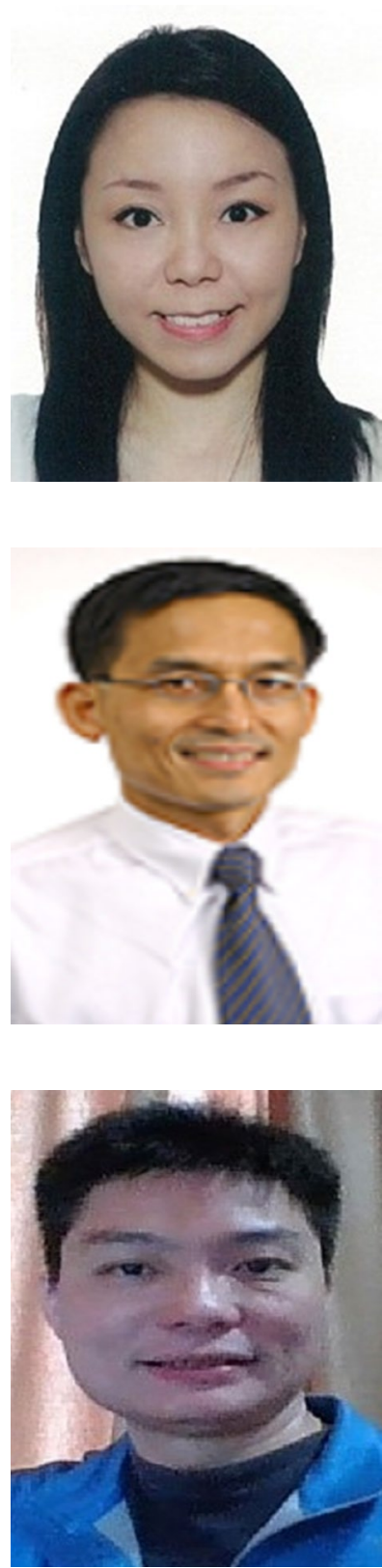

Vincent Lee is the Co-Principal Research Project Investigator. He works at SMRT Corporation Lab.

Yu Lian Wong is specialized in 2D and $3 \mathrm{D}$ graphic creation, as well as E-learning content development. She is experienced in producing quality digital contents for e-learning, gaming, virtual reality, advertising and print production industries in a professional environment.

Kevin Kho is Head of Collaborations at SMRT Trains Ltd and facilitated the inception and completion of the Virtual Reality Simulator and the associated Human Factors study. He has a B.Eng. in Civil Engineering, Master of Engineering (by Research) in Environmental Engineering from National University of Singapore, and M.Sc. in Biochemical Engineering from University College London. University. He received his B.Sc.

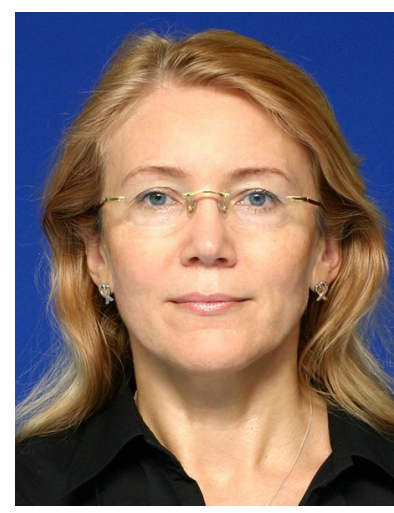

Olga Sourina is a Principal Research Scientist at Fraunhofer Singapore. She received M.Sc. in Computer Engineering from the Moscow Engineering Physics Institute, Russia, and Ph.D. from Nanyang Technological University, Singapore. Her research interest is in human factors, human-machine interaction, biosignals, machine learning and virtual reality.

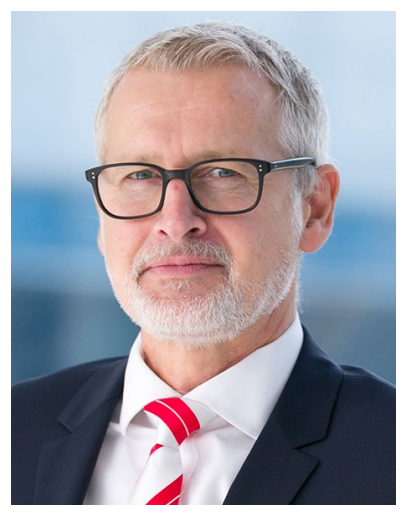

Wolfgang Mueller-Wittig currently heads Fraunhofer Singapore, the 8th subsidiary of the Fraunhofer-Gesellschaft and its very first in Asia. He is the Executive Director of Fraunhofer Centre for Interactive Digital Media and is an Adjunct Professor at the NTU School of Computer Science and Engineering. Prior to moving to Singapore in 2001, he worked as head of the "Visualisation Group" at Fraunhofer Institute for Computer Graphics Research in Germany (Fraunhofer IGD). He brings with him over 25 years of experience in industry-related research using visualization, virtual and augmented reality. 